\title{
Ventricular septal defect and tricuspid and mitral valve insufficiency caused by penetrating trauma
}

\author{
Ana Lopez-Marco', Jennifer Williams ${ }^{1}$, Christine Tan $^{2}$, Dheeraj Mehta ${ }^{1}$ \\ 'Department of Cardiothoracic Surgery, University Hospital of Wales, Cardiff CF14 4XW, UK. \\ ${ }^{2}$ Department of Anaesthesia, University Hospital of Wales, Cardiff CF14 4XW, UK.
}

\begin{abstract}
Correspondence to: Dr. Ana Lopez-Marco, Department of Cardiothoracic Surgery, University Hospital of Wales, Cardiff CF14 4XW, UK. E-mail: analopez1000@hotmail.com
\end{abstract} How to cite this article: Lopez-Marco A, Williams J, Tan C, Mehta D. Ventricular septal defect and tricuspid and mitral valve
insufficiency caused by penetrating trauma. Vesse/ Plus 2018;2:40. http://dx.doi.org/10.20517/2574-1209.2018.67

Received: 2 Oct 2018 First Decision: 16 Oct 2018 Revised: 17 Oct 2018 Accepted: 18 Oct 2018 Published: 5 Dec 2018

Science Editor: Mario F. L. Gaudino Copy Editor: Cui Yu Production Editor: Zhong-Yu Guo

\begin{abstract}
A 28-year old male sustaining a penetrating injury to the subxiphoid area presented to the emergency department fully conscious and haemodynamically stable. The CT scan revealed a localized infero-posterior pericardial collection. Emergency surgery was planned to evacuate the collection and assess the extent of injury. Intraoperative transoesophageal echocardiogram demonstrated severe tricuspid regurgitation due to transection of the papillary muscle, as well as a ventricular septal defect. Tricuspid repair with reconstruction of the papillary muscle, closure of the ventricular septal defect (VSD) and the right ventricular laceration was performed. Mitral regurgitation secondary to chordae rupture was identified following de-airing maneuvers, and subsequently underwent repair. Traumatic VSD and lesions of the mitral and tricuspid valves causing insufficiency have been reported before. They have been described in isolation or as combination of two lesions but never the combination of the three of them as described in this case.
\end{abstract}

Keywords: Cardiac trauma, penetrating wounds, emergency, ventricular septal defect, heart valve disease, transoesophageal echocardiography

\section{INTRODUCTION}

Penetrating cardiac injuries are usually secondary to stab or gunshot wounds. They represent a life threatening condition that often requires emergency surgery for evacuation of the commonly associated cardiac tamponade ${ }^{[1-6]}$. The free ventricular walls, especially on the right side are more commonly affected ${ }^{[1,5]}$. Injury of the cardiac valves and intraventricular septum is rare but it has been described, although more frequently associated with blunt cardiac trauma ${ }^{[1-6]}$.

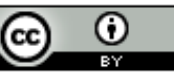

(C) The Author(s) 2018. Open Access This article is licensed under a Creative Commons Attribution 4.0 International License (https://creativecommons.org/licenses/by/4.0/), which permits unrestricted use, sharing, adaptation, distribution and reproduction in any medium or format, for any purpose, even commercially, as long as you give appropriate credit to the original author(s) and the source, provide a link to the Creative Commons license, and indicate if changes were made.

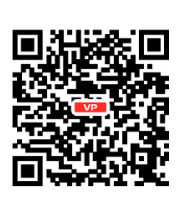


Traumatic ventricular septal defect (VSD) has been reported in 1\%-5\% of penetrating cardiac injuries. The diagnosis is usually obscured by the priority of stabilization and treatment of other more obvious and lethal injuries. It is consequently sometimes missed and diagnosed after the patient is recovered from the initial trauma ${ }^{[1-6]}$.

Mitral and tricuspid insufficiency secondary to cardiac trauma has also been reported, more commonly after blunt trauma. The trauma can potentially injure the valves at three different levels; papillary muscle, chordae and leaflet. Their diagnosis is also difficult, requiring an expert echocardiographer available at the time of the secondary survey. Otherwise, they are commonly missed until a full echocardiographic study is requested once the patient is more stable. High level of suspicion is key for their diagnosis ${ }^{[4-7]}$.

The gold-standard diagnosis for penetrating cardiac trauma is the echocardiography, to confirm haemopericardium and assess intracardiac structures. The VSD, however, can be missed initially due to muscle spasm or clot sealing the defect, consequently being commonly diagnosed and treated as a delayed complication of penetrating injuries ${ }^{[1-7]}$.

\section{CASE REPORT}

A 28-year-old male presented to the accident and emergency department following a stab injury to the anterior chest. He sustained a $6 \mathrm{~cm}$ stab wound in the subxiphoid area. On arrival, he was fully conscious and haemodynamically stable, with a heart rate of 90 beats $/ \mathrm{min}$, blood pressure $130 / 80 \mathrm{mmHg}$ and respiratory rate of 22 breaths/min. He was managed as per advanced trauma life support guidelines and being described as a "good responder" following commencement of fluid resuscitation.

Physical examination revealed a systolic murmur, multiple bruises to the forehead and sustained soft tissue swelling of the right orbit and face. Glasgow Comma Scale was 15/15 and his electrocardiogram revealed sinus tachycardia, with inversion of $\mathrm{T}$ wave in the inferior wall leads.

A thoracic and abdominal CT was done to assess the extent of the injuries. There was a mediastinal haematoma extending to the anterior cardiophrenic recess and moderate to large infero-posterior haemopericardium. The diaphragm remained intact [Figure 1].

He underwent a bedside transthoracic echocardiography (TTE) that reported preserved left ventricular function but with infero-posterior wall motion abnormality, causing tethering of the posterior leaflet of the mitral valve and therefore, moderate mitral regurgitation (MR). There was also moderate tricuspid regurgitation (TR) with pulmonary hypertension [pulmonary artery systolic pressure (PASP) $40 \mathrm{mmHg}+$ central venous pressure, normal PASP values $15-25 \mathrm{mmHg}$ ]; as well a $1 \mathrm{~cm}$ localized pericardial effusion in the infero-posterior wall, probably clotted. Right ventricular function was preserved.

He was therefore, prepared for emergency cardiac surgery to evacuate the haemopericardium and assess the extent of cardiac injuries. An intraoperative transoesophageal echocardiography (TOE) confirmed the infero-posterior pericardial collection as well as the inferior wall dyskinesia. There was severe TR due to rupture of the head of the posterior papillary muscle and a $2 \mathrm{~cm}$ VSD in the muscular interventricular septum causing a left-right shunt. The MR was mild-moderate and deemed to be secondary to the inferior wall dyskinesia. At this stage, there was no mitral prolapse [Figure 2A and B].

Surgery was performed through a median sternotomy, extending the stab wound superiorly. A clot was identified between the inferior wall and the diaphragm. As soon as the clot was dislodged, a profuse bleeding was identified from a $2 \mathrm{~cm}$ full thickness injury on the inferior wall of the right ventricle (RV). Control of the bleeding was achieved with manual pressure, whilst cardiopulmonary bypass (CPB) with bi-caval 


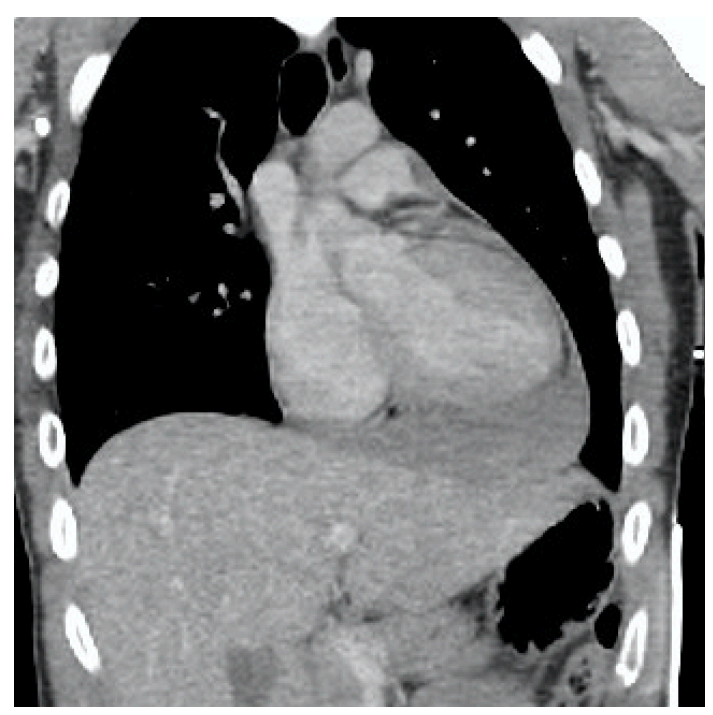

Figure 1. Preoperative CT scan of thorax and abdomen. Coronal view showing a localized collection in the inferior/posterior pericardial walls

cannulation and mild hypothermia was established. Myocardial protection was achieved with intermittent antegrade cardioplegia.

Following a right atriotomy, visualisation of the septum was achieved through the tricuspid valve (TV); there was a $3 \mathrm{~cm}$ VSD just below the posterior leaflet of the TV, which was detached due to complete transection of the papillary muscle head.

The VSD was closed with interrupted non-absorbable sutures reinforced with teflon. The papillary muscle head was re-implanted with a goretex suture. The TV was tested with saline test confirming competency. Closure of the right atrium was performed with a continuous $3 / 0$ prolene suture as well as the injury on the inferior wall of the RV using a continuous 3/0 prolene suture reinforced with 2 bands of teflon.

The patient was successfully removed from CPB. However the intraoperative TOE identified severe MR secondary to a new prolapse of A2 segment (or the central scallop of the anterior leaflet) with a flail chordae, which was probably partially ruptured and the complete transection occurred within the de-airing maneuvers [Figure 2C].

$\mathrm{CPB}$ was reinstituted and the left atrium (LA) was opened confirming the mitral aetiology; repair was performed with implantation of a goretex neochardae and a $30 \mathrm{~mm}$ physio II ring annuloplasty. The LA was closed and the CPB was weaned easily with good results of the repairs confirmed on TOE and with no residual interventricular shunt. After a long period to secure haemostasis, the chest was closed routinely after placing one mediastinal and one pericardial drains.

The postoperative period was satisfactory, being discharged by day 10 when the levels of oral anticoagulation were satisfactory. Follow-up visit at 6 weeks confirmed that he was asymptomatic and in sinus rhythm. The echocardiography confirmed a good result of the repairs, with trace TR, no MR and no residual VSD. The left and right ventricular functions were preserved with residual inferior hypokinesis of the RV.

\section{DISCUSSION}

Penetrating chest trauma, usually due to stab or gunshot wounds, can produce a wide variety of cardiac injuries which are life threatening in most cases. The mortality at the scene has been reported as high as $80 \%{ }^{[2]}$. 

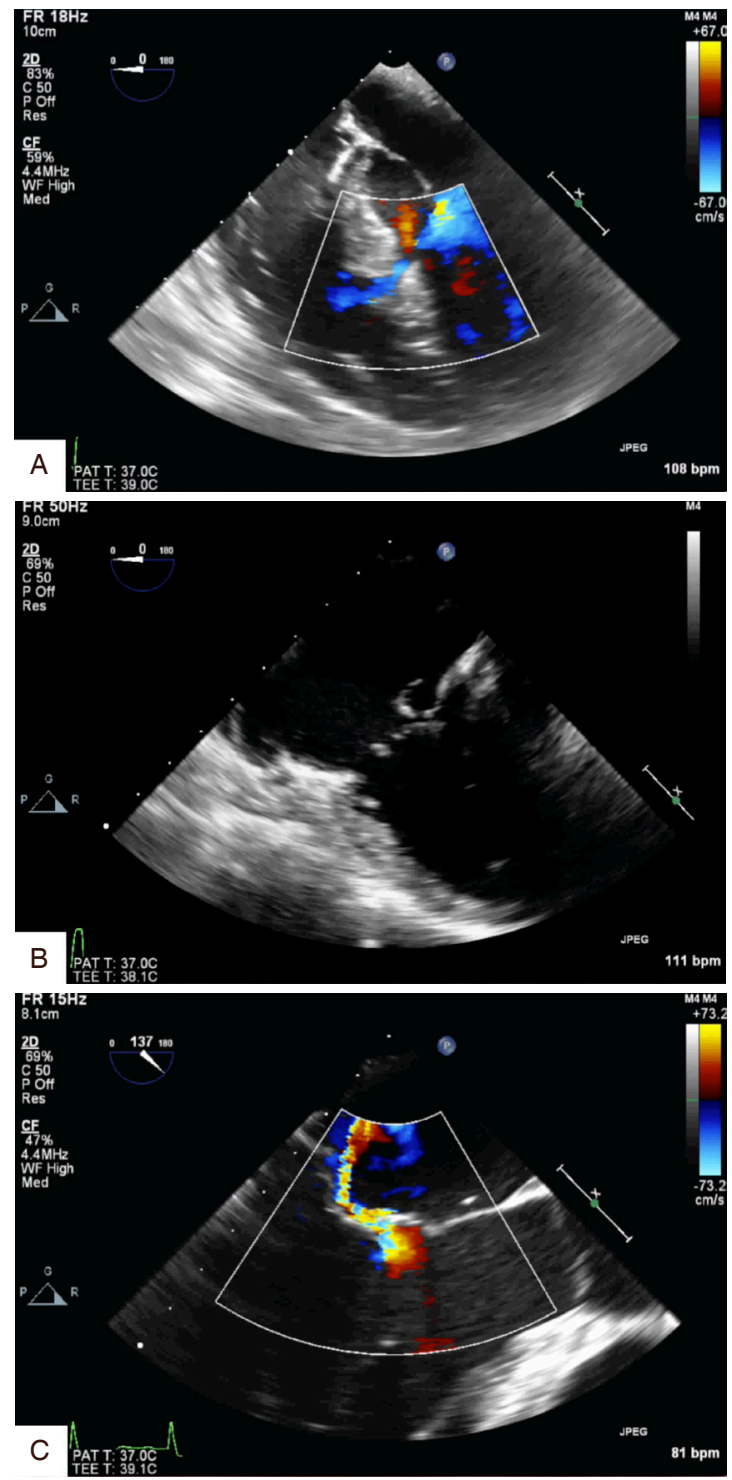

Figure 2. A: Intraoperative transoesophageal echocardiography (TOE) 4 chambers view with Doppler colour demonstrating the flow through the ventricular septal defect; B: intraoperative TOE, 4 chambers view, demonstrating the complete detachment of the tricuspid septal leaflet due to transection of the head of the papillary muscle; C: intraoperative TOE, apical long axis view with Doppler colour demonstrating the eccentric jet of mitral regurgitation caused by prolapse of A2 segment secondary to complete transection of the primary chordae

Of those who arrive in hospital, up to $70 \%$ survive to discharge, with rapid diagnosis and immediate treatment of injuries being the most important predictors of survival ${ }^{[2]}$. In addition to the free wall of the heart or the great arteries, intracardiac lesions affecting the valves or the septum is also possible. Traumatic VSD and lesions of the mitral and TVs causing insufficiency have been reported before. They have been described in isolation or as combination of two lesions but never the combination of the three of them as described in this case $e^{[1-7]}$.

The surgical treatment of penetrating cardiac injuries in an emergency is evacuation of the commonly associated cardiac tamponade and simple cardiorrhaphy of the lacerations on the external surface of the heart ${ }^{[1-7]}$. The subxiphoid approach for evacuation of the tamponade and lavage of the cavity has been reported as a safe alternative to full sternotomy for those cases who are haemodynamically stable and without active bleeding ${ }^{[8]}$. 


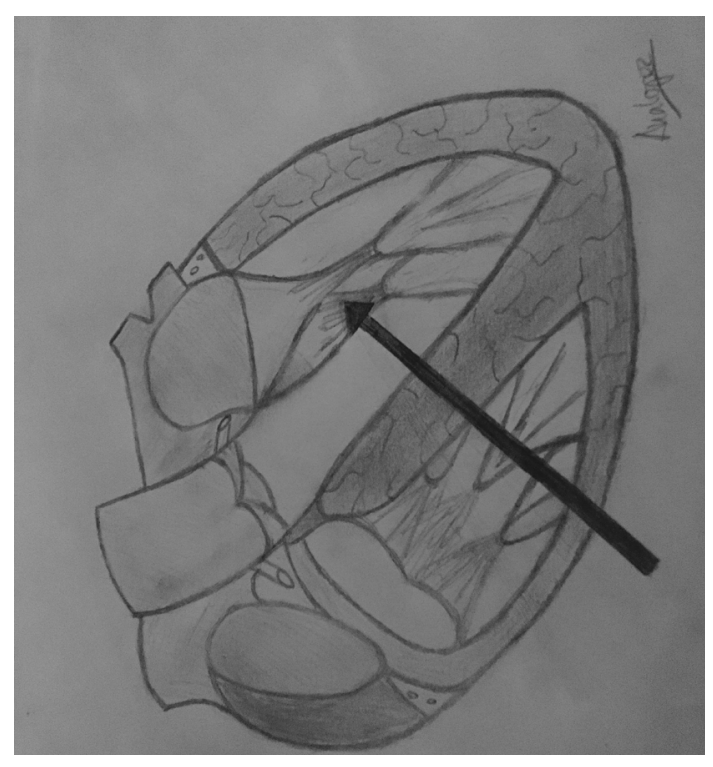

Figure 3. Drawing of a section of the heart showing the four chambers and postero-inferior walls. The thick arrow simulates the trajectory line of the stab injury to damage the inferior wall of the right ventricle, head of the papillary muscle supporting the posterior leaflet of the tricuspid valve, interventricular septum and chordae tendinae supporting A2 segment of the mitral valve

The gold-standard test for diagnosis in penetrating cardiac injuries is the echocardiography, which can confirm the presence of haemopericardium and assess the intra-cardiac structures. However, even in experienced hands, the diagnosis of intra-cardiac injuries, especially VSD can be obscured in this emergency setting. The VSD is often missed in the initial diagnosis due to focus on more threatening injuries or secondary to lack of intraoperative $\mathrm{TOE}^{[1-5]}$. They are therefore commonly diagnosed as a delayed complication of penetrating cardiac injuries and treated according to the associated symptoms ${ }^{[1-6]}$.

The atrioventricular valves can be injured during cardiac trauma at different levels: chordal rupture, papillary muscle rupture or leaflet defects. They are more frequently caused by blunt trauma, and their diagnosis can also be obscured during the initial evaluation. The degree of the valve insufficiency and the associated symptoms will determine the indication and timing for surgical intervention. Surgery in the early stages after the trauma favors the feasibility of valve repair ${ }^{[4-7]}$.

In our case, the TOE was the key in the prompt diagnosis of the three simultaneous lesions [Figure 3], allowing them to be repaired in a single operation. The fact that the patient was haemodynamically stable and with only a localized collection in the pericardium (confirmed by the CT and TTE) directed us towards an emergency operation for evacuation of the collection. We were inclined to start with a subxiphoid approach to evacuate the collection and perform a pericardial lavage to evaluate active bleeding but considered the intraoperative TOE necessary to further scrutinize the valve regurgitations addressed by the TTE.

The subxiphoid approach would not have been enough in our case, independent of the associated lesions. Active bleeding was identified as soon as the clotted collection was dislodged with the sucker, prompting a median sternotomy. It was then when the lesion on the free wall of the RV was seen, but decided to establish $\mathrm{CPB}$ as the VSD and valvular problems were already identified by the TOE at this point.

To summarize, penetrating cardiac trauma can cause a variety of lesions. Thorough examination with an early echocardiographic assessment should be mandatory for the evaluation of intracardiac injuries. Intraoperative TOE should always be performed in these cases as the TTE can often miss some of the intracardiac injuries. 


\section{DECLARATIONS}

\section{Authors' contributions}

Writing of the case report: Lopez-Marco A, Williams J

Consultant anaesthetist responsible for the case who also performed the intraoperative TOE and facilitated the images: Tan C

Operating consultant surgeon: Mehta D

\section{Availability of data and materials}

Not applicable.

\section{Financial support and sponsorship}

None.

\section{Conflicts of interest}

All authors declared that there are no conflicts of interest.

\section{Ethical approval and consent to participate}

The patient whom this case report refers gave his written consent form for publication of clinical information as well as diagnostic images.

\section{Consent for publication}

Not applicable.

\section{Copyright}

(c) The Author(s) 2018.

\section{REFERENCES}

1. Juneau D, Hermann D, Wells GL. Penetrating trauma resulting in ventricular septal defect. World J Cardiovasc Surg 2014;4:77-80.

2. Antoniades L, Petrou PM, Eftychiou C, Nicolaides E. A penetrating heart injury resulting in ventricular septal defect. Hellenic J Cardiol 2011;52:71-4.

3. Sugiyama G, Lau C, Tak V, Lee DC, Burack J. Traumatic ventricular septal defect. Ann Thorac Surg 2011;91:908-10.

4. Topaloglu S, Aras D, Cagli K, Ergun K, Deveci B, et al. Penetrating trauma to the mitral valve and ventricular septum. Tex Heart Inst J 2006;33:392-5.

5. Doty JR, Cameron DE, Elmaci T, Salomon NW. Penetrating trauma to the tricuspid valve and ventricular septum: delayed repair. Ann Thorac Surg 1999;67:252-3.

6. Shiber J, Cardarelli M. Traumatic ventricular septal defect and tricuspid regurgitation. J Emerg Med 2012;43:e141-2.

7. Ma WG, Luo GH, Sun HS, Xu JP, Hu SS, et al. Surgical treatment of traumatic tricuspid insufficiency: experience in 13 cases. Ann Thorac Surg 2010;90:1934-8.

8. Nicol AJ, Navsaria PH, Hommes M, Ball CG, Edu S, et al. Sternotomy or drainage for a haemopericardium after penetrating trauma: a randomized controlled trial. Ann Surg 2014;259:438-42. 